Mathematical Research Letters 3, 93-102 (1996)

\title{
HIGHER SPECTRAL FLOW
}

\author{
XianZhe Dai and Weiping Zhang
}

\begin{abstract}
For a continuous curve of families of Dirac type operators we define a higher spectral flow as a $K$-group element. We show that this higher spectral flow can be computed analytically by $\hat{\eta}$-forms, and is related to the family index in the same way as the spectral flow is related to the index. We also introduce a notion of Toeplitz family and relate its index to the higher spectral flow.
\end{abstract}

We introduce the notion of higher spectral flow, generalizing the usual spectral flow (cf [APS1]). The higher spectral flow is defined for a continuous one parameter family (i.e. a curve) of families of Dirac type operators parametrized by a compact space and is an element of the $K$-group of the parameter space. The (virtual) dimension of this $K$-group element is precisely the (usual) spectral flow. The definition makes use of the concept of spectral section introduced recently by Melrose-Piazza [MP].

We show that this higher version of spectral flow satisfies the basic properties of spectral flow. For example, its Chern character can be expressed analytically in terms of a generalization of the $\hat{\eta}$ form of Bismut-Cheeger [BC1]. The higher spectral flow is also related to the family index, in the same way as the spectral flow is related to the index.

We also introduce a notion of Toeplitz family and relate its index to the higher spectral flow. This generalizes a result of Booss-Wojciechowski [BW, Theorem 17.17]. Finally we use higher spectral flow to prove a generalization of the family index theorem for manifolds with boundary [BC2], [BC3], [MP].

The details and the proofs will appear in [DZ2].

\section{Spectral flow and spectral section}

We take the definition of spectral flow as in [APS1]. Thus, if $D_{s}, 0 \leq$ $s \leq 1$, is a curve of self-adjoint Fredholm operators, the spectral flow

Received October 12, 1995.

1991 Mathematics Subject Classification. Primary 58XX.

The first author is partially supported by NSF Grant DMS9204267 and Alfred P. Sloan Fellowship

The second author is partially supported by the Chinese National Science Foundation and the State Education Commission of China. 
$s f\left\{D_{s}\right\}$ counts the net number of eigenvalues of $D_{s}$ which change sign when $s$ varies from 0 to 1 . (Throughout the paper a family always means a continuous family, and a curve always means a one parameter family.)

The notion of spectral section can be defined for a family of self adjoint first order elliptic pseudodifferential operators [MP].

Definition. A spectral section for a family of self adjoint first order elliptic pseudodifferential operators $D_{z}(z \in B)$ is a family of self adjoint pseudodifferential projections $P_{z}$ such that for some smooth function $R: B \longrightarrow \mathbb{R}$ and every $z \in B$

$$
D_{z} u=\lambda u \Rightarrow\left\{\begin{array}{l}
P_{z} u=u \text { if } \lambda>R(z) \\
P_{z} u=0 \text { if } \lambda<-R(z) .
\end{array}\right.
$$

As is proved in $[\mathrm{MP}]$, when the parameter space is compact, the existence of a spectral section is equivalent to the vanishing of the (analytic) index of the family. Thus the existence for a one parameter family is always assured.

Now let $D_{s}$ be a curve of self adjoint elliptic pseudodifferential operators. Let $Q_{s}$ be the spectral projection onto the direct sum of eigenspaces of $D_{s}$ with nonnegative eigenvalues (the APS projection). The following theorem provides a link between the above two notions.

Theorem 1.1. Let $P_{s}$ be a spectral section of $D_{s}$. Then $\left[Q_{1}-P_{1}\right]$ defines an element of $K^{0}(p t) \cong \mathbb{Z}$ and so does $\left[Q_{0}-P_{0}\right]$. Moreover the difference $\left[Q_{1}-P_{1}\right]-\left[Q_{0}-P_{0}\right]$ is independent of the choice of the spectral section $P_{s}$, and it computes the spectral flow of $D_{s}$ :

$$
s f\left\{D_{s}\right\}=\left[Q_{1}-P_{1}\right]-\left[Q_{0}-P_{0}\right] .
$$

Proof. The independence of the choice of spectral section follows from a construction in [MP, Proposition 2]. For (1.1), we use spectral sections to reduce it to the finite dimensional case, where it can be easily verified.

This leads us to the notion of

\section{Higher spectral flow}

Let $\pi: X \longrightarrow B$ be a smooth fibration with the typical fiber $Z$ an odd dimensional closed manifold and $B$ compact. A family of self adjoint elliptic pseudodifferential operators on $Z$, parametrized by $B$, will be called a $B$-family. Consider a curve of $B$-families, $D_{u}=\left\{D_{b, u}\right\}, u \in[0,1]$.

Assuming that the index bundle of $D_{0}$ vanishes, the homotopy invariance of the index then implies that the index bundle of each $D_{u}$ vanishes. Let $Q_{0}, Q_{1}$ be spectral sections of $D_{0}, D_{1}$ respectively. If we consider the total family $\tilde{D}=\left\{D_{b, u}\right\}$ parametrized by $B \times I$, then there is a total spectral section $\tilde{P}=\left\{P_{b, u}\right\}$. Let $P_{u}$ be the restriction of $\tilde{P}$ over $B \times\{u\}$. 
According to [MP] difference of spectral sections defines $K$-group element of the parameter space.

Definition. The (higher) spectral flow $s f\left\{\left(D_{0}, Q_{0}\right),\left(D_{1}, Q_{1}\right)\right\}$ between the pairs $\left(D_{0}, Q_{0}\right),\left(D_{1}, Q_{1}\right)$ is an element in $K(B)$ defined by

$$
\operatorname{sf}\left\{\left(D_{0}, Q_{0}\right),\left(D_{1}, Q_{1}\right)\right\}=\left[Q_{1}-P_{1}\right]-\left[Q_{0}-P_{0}\right] \in K(B) .
$$

The definition is independent of the choice of the (total) spectral section $P$, as it follows again from [MP]. When $D_{u}, u \in S^{1}$ is a periodic family, we choose $Q_{1}=Q_{0}$. In this case the spectral flow turns out to be independent of $Q_{0}=Q_{1}$ and therefore defines an invariant of the family, denoted $s f\left\{D_{u}\right\}$.

For the most part of this paper we are going to restrict our attention to $B$-families of Dirac type operators, defined as follows. For simplicity we assume that the vertical tangent bundle $T Z \longrightarrow X$ is spin and carries a fixed spin structure ${ }^{1}$. Let $g^{T Z}$ be a metric on $T Z$. Let $E$ be a complex vector bundle over $X$ with an hermitian metric $g^{E}$ and compatible connection $\nabla^{E}$. Corresponding to these geometric data we have a family of Dirac operators $D_{b}^{E}, b \in B$. This is a family of self adjoint elliptic operators on $Z$ parametrized by $B$.

Definition. By a $B$-family of self adjoint Dirac type operators we mean a family of self adjoint elliptic operators $\tilde{D}_{b}$ parametrized by $B$ whose principal symbol is the same as that of $D_{b}^{E}$.

Basic assumption. We assume that the family $D_{b}^{E}$ has vanishing index bundle.

A typical example satisfying our basic assumption is the family of signature operators. More generally a $B$-family whose kernels have constant dimension will always satisfy the basic assumption. Another class of examples comes from the boundary family of a family of Dirac operators on manifolds with boundary.

Now we can speak of the higher spectral flow of a curve of $B$-families of Dirac type operators, given the basic assumption.

\section{3. $\hat{\eta}$-form and higher spectral flow}

By $[\mathrm{MP}]$, given a spectral section $P=\left\{P_{b}\right\}$ of a $B$-family $D=\left\{D_{b}\right\}$, there is a family of zeroth order finite rank pseudodifferential operators $A=\left\{A_{b}\right\}$ such that each $\tilde{D}_{b}=D_{b}+A_{b}$ is invertible and that $P_{b}$ is precisely the APS projection of $\tilde{D}_{b}$. (We will call $A$ a Melrose-Piazza operator associated to the spectral section.)

\footnotetext{
${ }^{1}$ Our discussion extends without difficulty to the more general case when there are smoothly varying $\mathbb{Z}_{2}$-graded Hermitian Clifford modules over the fibers, with graded unitary connections.
} 
For our purpose we define a $\hat{\eta}$-form which generalizes both [BC1] and $[\mathrm{MP}]$. The main point is that we need to use general superconnections, not just Bismut superconnection [B]. We first introduce some notations.

Let $X$ be the total space of the fibration whose typical fiber is $Z$ and the base $B$. Choosing a connection amounts to a splitting

$$
T X=T Z \oplus T^{H} X \text {. }
$$

We also have the identification $T^{H} X=\pi^{*} T B$.

Endow $B$ with a metric $g^{T B}$ and let $g^{T X}$ be the metric defined by

$$
g^{T X}=g^{T Z} \oplus \pi^{*} g^{T B} .
$$

Let $P, P^{\perp}$ be the orthogonal projections of $T X$ onto $T Z, T^{H} X$ respectively and denote by $\nabla^{T X}, \nabla^{T B}$ the Levi-Civita connections. Following Bismut [B], let $\nabla^{T Z}$ be the connection on the vertical bundle defined by $\nabla^{T Z}=P \nabla^{T X} P$. This is a connection compatible with the metric $g^{T Z}$ and independent of the choice of the metric $g^{T B}$.

We use $S(T Z)$ to denote the spinor bundle of $T Z$. Then the connection lifts to a connection on the spinor bundle. Also following Bismut we view $\Gamma(S(T Z) \otimes E)$ as the space of sections of an infinite dimensional vector bundle $H_{\infty}$ over $B$, with fiber

$$
H_{\infty, b}=\Gamma\left(S\left(T Z_{b}\right) \otimes E_{b}\right) .
$$

Then $\nabla^{S(T Z) \otimes E}$ determines a connection on $H_{\infty}$ by the prescription:

$$
\tilde{\nabla}_{X} h=\nabla_{X^{H}} h
$$

Now, let $\left\{D_{b}\right\}$ be a $B$-family of self adjoint Dirac type operators as defined previously, and $A$ a Melrose-Piazza operator associated to a spectral section $P$.

Definition. For any $B_{i} \in \Omega^{i}\left(T^{*} B\right) \otimes \Gamma(\operatorname{End}(S(T Z) \otimes E))=\Omega^{i}\left(T^{*} B\right) \otimes$ $c l(T Z) \otimes \operatorname{End}(E)$ odd, and any $t>0$, we define the superconnection $B_{t}$ to be

$$
B_{t}=\tilde{\nabla}+\sqrt{t}(D+\rho(t) A)+\sum_{i=0}^{\operatorname{dim} B} t^{\frac{1-i}{2}} B_{i},
$$

where $\rho$ is a cut-off function in $t: \rho(t)=1$ when $t>8$ and $\rho(t)=0$ when $t<2$.

Definition. We define, for $\Re(s) \gg 0$,

$$
\hat{\eta}(P, A, s)=\frac{1}{2 \sqrt{\pi}} \int_{0}^{\infty} t^{\frac{s}{2}} \operatorname{Tr}^{\text {even }}\left[\frac{d B_{t}}{d t} e^{-B_{t}^{2}}\right] d t .
$$

This defines a differential form on $B$ which depends holomorphically on $s$ for $\Re(s) \gg 0$. By the standard argument it extends to a meromorphic 
function of $s$ in the whole complex plane with only simple poles. Note that our definition has an extra factor of $\frac{1}{2}$.

Theorem 3.1. The residue of $\hat{\eta}(P, A, s)$ at $s=0$ is exact.

Definition. The $\hat{\eta}$-form is defined as

$$
\hat{\eta}(D, P)=\left\{\hat{\eta}(P, A, s)-\frac{\operatorname{Res}_{s=0}\{\hat{\eta}(P, A, s)\}}{s}\right\}_{s=0} .
$$

Remark. The $\hat{\eta}$-form for a general superconnection is defined in [BC1] in the finite dimensional case.

A variational argument shows

Proposition 3.2. The value of $\hat{\eta}(D, P)$ in $\Omega^{*}(B) / d \Omega^{*}(B)$ is independent of the choice of the cut-off function $\rho$ and the Melrose-Piazza operator A.

The dependence of $\hat{\eta}$-form on the spectral section is also well understood. The following is a slight generalization of a result of [MP].

Theorem 3.3. If $P_{0}$ and $P_{1}$ are spectral sections of the family $D$, then their difference defines an element $\left[P_{1}-P_{0}\right]$ in $K(B)$. Moreover

$$
\operatorname{ch}\left(P_{1}-P_{0}\right)=\hat{\eta}\left(D, P_{1}\right)-\hat{\eta}\left(D, P_{0}\right) \text { in } H^{*}(B) .
$$

The following theorem generalizes the well known relationship between the spectral flow and the eta invariant.

Theorem 3.4. Let $D_{u}$ be a curve of $B$-families of Dirac type operators and $Q_{0}, Q_{1}$ spectral sections of $D_{0}, D_{1}$ respectively. Let $\tilde{B}_{t}(u)=\tilde{\nabla}+$ $\sqrt{t}\left(D_{u}+\rho(t) \tilde{A}\right)+\sum_{i \geq 1} t^{\frac{1-i}{2}} B_{i}(u)$ be a curve of superconnections. Then we have the following identity in $H^{*}(B)$ :

$\operatorname{ch}\left(\operatorname{sf}\left\{\left(D_{0}, Q_{0}\right),\left(D_{1}, Q_{1}\right)\right\}\right)=\hat{\eta}\left(D_{1}, Q_{1}\right)-\hat{\eta}\left(D_{0}, Q_{0}\right)-\int_{0}^{1} \frac{d \hat{\eta}\left(D_{u}, P_{u}\right)}{d u} d u$,

where the last term is a local invariant computable from the asymptotic expansion

$$
\left.\frac{1}{\sqrt{\pi}} \frac{\partial}{\partial s}\left\{\operatorname{Tr}^{\text {even }}\left[\exp \left(-\tilde{B}_{t}^{2}(u)-s \frac{\partial \tilde{B}_{t}(u)}{\partial u}\right)\right]\right\}\right|_{s=0}=\sum_{i \geq-k} a_{i} t^{i}
$$

i.e., we have

$$
\frac{d \hat{\eta}\left(D_{u}, P_{u}\right)}{d u}=a_{0}(u)
$$

This theorem provides a way to compute, analytically, the Chern character of higher spectral flow. We will use Formula (3.4) to compute the higher spectral flow of both a periodic family and a Toeplitz family. 


\section{Higher spectral flow and family index}

We consider a periodic family $D_{b, u}, b \in B, u \in S^{1}$ of $B$-families of Dirac type operators, where $B$ is a closed manifold. The total family $\tilde{D}_{b, u}$ is a family of Dirac operators on an even dimensional space (the total space of a fibration over $S^{1}$ with the typical fiber $Z$ ). Thus it defines an index bundle $\operatorname{ind}(\tilde{D}) \in K(B)$. The following theorem generalizes the well known relationship between the spectral flow and the index.

Theorem 4.1. We have

$$
\operatorname{ch}(\operatorname{ind}(\tilde{D}))=\operatorname{ch}\left(s f\left\{D_{u}\right\}\right) \text { in } H^{*}(B) .
$$

Proof. We apply Theorem 3.4 to compute the higher spectral flow and show that the result agrees with the Atiyah-Singer formula [AS] for the family index.

Remark. It is very likely that the higher spectral flow and the family index actually equal as $K$-group elements, but at the moment we do not see how to prove this.

As a consequence, we deduce the following relation between the spectral flow and higher spectral flow.

Corollary 4.2. Let $D_{u}^{Z}$ be a periodic family of B-families of Dirac operators and let $D_{u}^{X}$ be the one parameter family of the total Dirac operators. Then we have

$$
s f\left\{D_{u}^{X}\right\}=\int_{B} \hat{A}(B) \operatorname{ch}\left(\operatorname{sf}\left\{D_{u}^{Z}\right\}\right) .
$$

\section{Higher spectral flow and Toeplitz family}

Now let $\mathbb{C}^{N}$ be a trivial vector bundle over $X$ with its canonical (trivial) metric and connection. Let

$$
g: X \longrightarrow \mathrm{GL}(N, \mathbb{C}) .
$$

Then $g$ acts on the trivial bundle. Moreover it extends in the obvious way to an operator

$$
g_{b}: \mathrm{L}^{2}\left(S\left(T Z_{b}\right) \otimes E_{b}\right) \otimes \mathbb{C}^{N} \longrightarrow \mathrm{L}^{2}\left(S\left(T Z_{b}\right) \otimes E_{b}\right) \otimes \mathbb{C}^{N}
$$

Let $P$ be a spectral section of the $B$-family $D$. Then $P$ also extends to an operator

$$
P_{b}: \mathrm{L}^{2}\left(S\left(T Z_{b}\right) \otimes E_{b}\right) \otimes \mathbb{C}^{N} \longrightarrow \mathrm{L}^{2}\left(S\left(T Z_{b}\right) \otimes E_{b}\right) \otimes \mathbb{C}^{N}
$$

by acting as identity on the factor $\mathbb{C}^{N}$. Let $L_{P, b}$ be the image space of $\mathrm{L}^{2}\left(S\left(T Z_{b}\right) \otimes E_{b}\right) \otimes \mathbb{C}^{N}$ under $P_{b}$. 
Definition. For any $b \in B$, define $T(g)_{b}$ to be the bounded linear operator

$$
T(g)_{b}=P_{b} g_{b}: L_{P, b} \longrightarrow L_{P, b} .
$$

This is a generalization of the notion of Toeplitz operator. $T(g)$ is a continuous family of Fredholm operators parametrized by $B$, and by Atiyah-Singer [AS], it defines an element

$$
\operatorname{ind}_{a}(T(g)) \in K(B),
$$

its index bundle. The index bundle of the Toeplitz family does not depend on the geometric data and the spectral section.

Without loss of generality, we now assume that $g$ is unitary. Extend $D$ to $\mathrm{L}^{2}\left(S\left(T Z_{b}\right) \otimes E_{b}\right) \otimes \mathbb{C}^{N}$ in the obvious way. Then $P$ is still a spectral section for $D$. Moreover, $g P g^{-1}$ is a spectral section for $g D g^{-1}$. Connecting $D$ and $g D g^{-1}$ by the linear path, we have the following generalization of [BW, Theorem 17.17].

Theorem 5.1. We have an equality

$$
\operatorname{ch}\left(\operatorname{ind}_{a}(T(g))\right)=\operatorname{ch}\left(s f\left\{(D, P),\left(g D g^{-1}, g P g^{-1}\right)\right\}\right) .
$$

Remark. In this theorem one can verify directly that the right hand side does not depend on $P$.

Proof. Once again we apply Theorem 3.4 to compute the higher spectral flow. On the other hand one can still use the argument of [BD] to compute the family index of the Toeplitz family. The computations show that they are equal. We point out that we shall use a special modification of the Bismut superconnection to make sure that the local index type calculation can be carried out.

As an interesting example we can take $D$ a $B$-family of signature operators. In this case the higher spectral flow in Theorem 5.1 is usually nontrivial, although the higher spectral flow of a one parameter family of $B$-families of signature operators is always zero.

Theorem 5.1 is in fact true in $K$-theory, see [DZ2] for detail ${ }^{2}$.

\section{An extension of family index theorem}

An important property of the spectral flow is that it measures the change of the index for manifolds with boundary under continuous deformation [DZ1]. We show that higher spectral flow measures the change of the family index for manifolds with boundary under continuous deformation.

\footnotetext{
${ }^{2}$ We thank Krysztof Wojciechowski for helpful discussions.
} 
Let $\pi: M \longrightarrow B$ be a smooth fibration with the typical fiber $Y$ an even dimensional manifold with boundary $Z$. For simplicity ${ }^{3}$ we assume that the vertical tangent bundle $T Y \longrightarrow M$ is spin and carries a fixed spin structure. Let $g^{T Y}$ be a metric on $T Y$ which is of product type near the boundary. Let $E$ be a complex vector bundle over $M$ with an hermitian metric $g^{E}$ and compatible connection $\nabla^{E}$. Corresponding to these geometric data we have a family of Dirac operators $D_{b}^{E}, b \in B$. This is a family of elliptic operators on the manifold with boundary $Y$, parametrized by $B$.

Definition. By a $B$-family of Dirac type operators on the manifold with boundary $Y$ we mean a family of elliptic operators $\tilde{D}_{b}$ parametrized by $B$ which is of product type near the boundary and whose principal symbol is the same as that of $D_{b}^{E}$. Moreover, we assume that the boundary family is also a $B$-family of Dirac type operators.

It follows that spectral sections always exist for the boundary family. Choosing a spectral section, we can then define the family index for this $B$-family of Dirac type operators on the manifold with boundary as in $[\mathrm{MP}]$.

Theorem 6.1. Let $\tilde{D}_{b, u}$ be a one parameter family of B-families of Dirac type operators on the manifold with boundary $Y$. Let $\tilde{D}_{b, u}^{\partial}$ denote the boundary family. Let $Q_{b, 0}, Q_{b, 1}$ be spectral sections for $\tilde{D}_{b, 0}^{\partial}, \tilde{D}_{b, 1}^{\partial}$ respectively. Then

$$
\operatorname{ind}\left(\tilde{D}_{1}, Q_{1}\right)-\operatorname{ind}\left(\tilde{D}_{0}, Q_{0}\right)=-s f\left\{\left(\tilde{D}_{0}^{\partial}, Q_{0}\right),\left(\tilde{D}_{1}^{\partial}, Q_{1}\right)\right\} \text { in } K(B) .
$$

This is a generalization of [DZ1, Theorem 1.1]. Using this result we can prove the following generalization of the family index theorem for manifolds with boundary [BC2], [BC3], [MP].

Theorem 6.2. Let $\tilde{D}$ be a B-family of Dirac type operators on the manifold with boundary $Y$ and $\tilde{D}^{\partial}$ its boundary family. Let $Q$ be a spectral section of $\tilde{D}^{\partial}$. Then

$$
\operatorname{ch}(\operatorname{ind}(\tilde{D}, Q))=\int_{Y} a_{0}-\hat{\eta}\left(\tilde{D}^{\partial}, Q\right),
$$

where $a_{0}$ is the constant term in the asymptotic expansion of

$$
\operatorname{Tr}_{s}\left[\exp \left(-\tilde{B}_{t}^{2}\right)\right]=\sum_{i \geq-\frac{\operatorname{dim} Y}{2}-\left[\frac{\operatorname{dim} B}{2}\right]} a_{i} t^{i},
$$

\footnotetext{
${ }^{3}$ Once again our discussion extends to the more general case.
} 
with an arbitrary total superconnection $\tilde{B}_{t}$ now extended to the double of the fibration, while $\hat{\eta}\left(\tilde{D}^{\partial}, Q\right)$ is the $\hat{\eta}$-form defined using the boundary superconnection induced from $\tilde{B}_{t}$.

Proof. We use the family index theorem of $[\mathrm{MP}]$ for the Dirac family and flow to the more general Dirac type family.

Remark. This is the analogue in the family case for the general index formula of Atiyah-Patodi-Singer for manifold with boundary [APS2].

\section{Acknowledgement}

Part of this work is done while the first author was visiting the Nankai Institute of Mathematics. He is very grateful to the Chinese National Science Foundation for partial financial support and the Nankai Institute for its hospitality. The second author would like to thank the Courant Institute for its hospitality. Both authors thank the referee for very helpful suggestions and comments.

\section{References}

[APS1] M.F.Atiyah, V.K.Patodi and I.M.Singer, Spectral asymmetry and Riemannian geometry III. Proc. Cambridge Philos. Soc. 79(1976), pp 71-99.

[APS2] _ Spectral asymmetry and Riemannian geometry I. Proc. Cambridge Philos. Soc. 77(1975), pp 43-69.

[AS] M.F.Atiyah and I.M.Singer, The index of elliptic operators IV, Ann. of Math., 93(1971), pp 119-138.

[B] J.-M.Bismut, The Atiyah-Singer index theorem for a family of Dirac operators: two heat equation proofs, Invent. Math., 83(1986), pp 91-151.

[BC1] J.-M.Bismut and J.Cheeger, $\eta$-invariants and their adiabatic limits, J. of Amer. Math. Soc., 2(1989), pp 33-70.

[BC2] _ Families index theorem for manifolds with boundary I, II, J. Functional Analysis, 89(1990), pp 313-363 and 90(1990), pp 306-354.

[BC3] _ Remarks on the index theorem for families of Dirac operators on manifolds with boundary, in Differential Geometry, B. Lawson and K. Tenenblat (eds.), Longman Scientific, 1992, pp 59-84.

[BD] P. Baum and R. Douglas, Toeplitz operators and Poincare duality, in Proceedings of the Toeplitz memorial conference, I. C. Gohberg (ed.), Birkhäuser, 1981, pp 137-166.

[BW] B. Booss and K. Wojciechowski, Elliptic boundary problems for Dirac operators, Birkhäuser, 1993. 
[DZ1] X. Dai and W. Zhang, The Atiyah-Patodi-Singer index theorem: a proof using embeddings, C. R. Acad. Sci. Paris, Series I, 319(1994), pp 1293-1297.

[DZ2] _ , in preparation

[MP] R. B. Melrose, P. Piazza Families of Dirac operators, boundaries and the $b$ calculus, to appear in J. Diff. Geom.

Department of Mathematics, University of Southern California, Los AnGLES, CA 90089

E-mail address: xdai@math.usc.edu

Nankai Institute of Mathematics, Tianjin, 300071, P.R. China

Current address: Courant Institute, NYU, New York, NY 10012

E-mail address: weiping@math1.cims.nyu.edu 